\title{
La politique royale normande et les comtés calabrais dans la seconde moitié du XII ${ }^{\mathrm{e}}$ siècle
}

\author{
L'apport du fonds de S. Stefano del Bosco
}

\author{
Annick Peters-Custot
}

La conquête normande de l'Italie méridionale, au XI ${ }^{\text {e }}$ siècle, a fait émerger au milieu du XII ${ }^{\text {e }}$ siècle une monarchie dont l'originalité, au sein de l'espace occidental, a pu préfigurer la royauté, considérée comme hors norme, de Frédéric II. Cette originalité ne doit pas masquer les divergences territoriales liées aux modalités assez disparates de cette conquête, relativement anarchique dans les zones les plus septentrionales, et au contraire centralisée en Calabre et en Sicile dans les mains des membres les plus éminents d'une famille normande, les Hauteville. Ainsi, par la suite, le mode d'organisation et de gestion administratives des territoires maintint, même après la conquête de la Pouille par Roger II, cette dichotomie issue de la conquête, et que vinrent renforcer les héritages administratifs des régimes antérieurs : en particulier, Sicile et Calabre méridionale furent bientôt associées tant par les pratiques administratives et par les ressorts des institutions territoriales, que par le maintien d'une forme plus centralisée du pouvoir, dans les mains du souverain.

Prennent place dans ce système politique et administratif normand, présent dans la Calabre méridionale : le monopole des Hauteville sur les forteresses, l'originalité de l'introduction de la féodalité, le rôle du souverain sur son Église, et les modalités de distributions des terres, fiefs et droits. Rappelons que le comte, puis le roi de Sicile ont revendiqué et maintenu, y compris dans les divers

1. Voir Fodale 1970, rééd. dans Fodale 1991. Je me permets de renvoyer également à Peters-Custot 2009, p. 234-246 ainsi qu'à Peters-Custot 2007, p. 57-77.

2. Peters-Custot 2009, p. 308-309.

3. Catalogus Baronum 1972; Cuozzo 1972.

4. Cahen 1940, p. 58-61. traités successifs signés avec la papauté (Bénévent, Gravina), une mainmise importante sur l'organisation de l'Église, surtout en Sicile et en Calabre, protégée durablement de l'ingérence de Rome dans les institutions et nominations ecclésiastiques $^{1}$. Dans la Calabre, qui avait ignoré à l'époque byzantine (comme la Pouille byzantine du reste ${ }^{2}$ ) toute forme de privatisation de l'autorité publique, les Normands imposèrent un régime féodal très contrôlé dès l'époque comtale, avant même une normalisation juridique qui, sous la royauté, imposa à tout le royaume une féodalité essentiellement administrative et militaire, et à tout le moins fortement domestiquée, dont témoigne, pour l'Italie normande à l'exclusion de la Sicile et de la Calabre, le Catalogue des Barons ${ }^{3}$. Parallèlement, le comte de Sicile organisa la distribution des fiefs, en conservant une partie considérable, voire majoritaire des terres sous son contrôle direct dans le domaine comtal (puis royal). Dans la Calabre méridionale en particulier, seuls des fiefs de petite taille sont attestés dans les sources, et une bonne partie d'entre eux est intégrée dans le domaine royal après la répression des révoltes sous Roger $\mathrm{II}^{4}$. Même si des fiefs confiés à des représentants de l'immigration normande sont mentionnés dans la documentation calabraise dans la seconde moitié du XII ${ }^{e}$ siècle ${ }^{5}$, il reste que la féodalité calabraise est soumise au souverain et dépendante de celui-ci pendant toute l'époque

\footnotetext{
5. En 1188, le fief d'Asklettin de Brix fait l'objet d'une enquête pour déterminer ses limites, droits et possessions. Il avait été concédé en 1138 par le roi, et est ainsi confirmé, avec ses terres et ses "hommes" (bellanoi/villani) : Trinchera 1865, $n^{\circ} 225$.
} 
normande. Par ailleurs, la seigneurie banale est, dans la Calabre méridionale, pratiquement absente: si la seigneurie foncière a pu s'intégrer d'autant plus facilement dans le paysage calabrais qu'elle s'est appuyée sur le socle matériel constitué par l'ancienne grande propriété byzantine, celle des proastéia ${ }^{6}$, en revanche il est rare que les seigneurs, tant monastiques que laïcs, obtiennent des bribes d'autorité publique. Le souverain maintient donc les droits publics comme monopoles régaliens, et compense le manque à gagner par de larges concessions de terres et d'hommes faites aux seigneurs (notamment monastiques ou ecclésiastiques) ${ }^{7}$. Par ailleurs, pour ce qui est des églises et des monastères, des concessions de dîmes sur les revenus publics viennent compenser l'absence de dîmes sur les revenus privés ${ }^{8}$.

Tous ces faits bien connus ont une incidence sur l'organisation des comtés calabrais, que JeanMarie Martin a décrite dans une étude générale dont nous reprendrons ici les conclusions ${ }^{9}$ en essayant de les préciser par l'examen d'un fonds d'archives peu utilisé, celui des actes grecs de S. Stefano del Bosco.

\section{LES COMTÉS CALABRAIS}

\section{À L'ÉPOQUE NORMANDE}

Dans la Calabre méridionale, les comtés sont peu nombreux et tardifs, la première attestation sûre d'un comté, celui de Catanzaro, remontant aux années 1131-1132, soit immédiatement après la fondation du royaume par Roger $\mathrm{II}^{10}$. Ce comté de Catanzaro est d'ailleurs à la fois le premier et le seul qui ait un peu de consistance: d'autres comtés calabrais sont attestés au début du XIII $^{e}$ siècle (comme à San Marco Argentano en 1205, à Crotone vers 1204-1208), mais leur multiplication correspond à des créations spontanées et autoproclamées, liées à la période anarchique de la minorité de Frédéric II, et non à des décisions royales $^{11}$. Si le titre de comte de Catanzaro est attesté en 1132, dans la titulature du premier titulaire, Geoffroy de Loritello, ce dernier expose que son père Raoul, mort avant 1111, portait également le titre de comte ${ }^{12}$. Ce comté de Catanzaro est donc vraisemblablement créé par le roi Roger II en transformant en comté, en 1132 ou peu avant, la seigneurie de Catanzaro, que tenait depuis longtemps une branche de la famille comtale de Loritello. À la seigneurie de Catanzaro était rattachée, vraisemblablement dès l'origine, celle de Badolato $^{13}$.

Jusque vers 1154, c'est le seul comté attesté dans la Calabre méridionale, fermement gouvernée par le roi normand. Il subit des évolutions complexes, qui ne sont souvent attestées que de manière indirecte dans les sources, au gré des suscriptions ou des mentions diplomatiques et des passages plus ou moins précis des chroniques. En particulier, on s'aperçoit que le comté de Catanzaro a paru suffisamment dangereux ou menaçant pour que, malgré sa topographie d'emblée
6. La grande propriété d'époque byzantine en Calabre est surtout attestée pour les cathédrales et monastères, comme l'attestent quelques fonds d'archives des évêchés byzantins, tels le cartulaire d'Oppido ou le brébion (inventaire des biens et redevances) de la métropole de Reggio. Voir Brébion 1974 et La Théotokos de Hagia-Agathè 1972. Toutefois, la fortune foncière de grandes familles italo-grecques d'origine byzantine à l'époque normande implique des antécédents byzantins (Peters-Custot 2009, p. 160-161 et p. 402-409).

7. Martin 2008, p. 177-206 et Martin 1999, p. 227-259.

8. Toomaspoeg 2009; pour les corrections à apporter concernant l'époque normande, voir la recension en ligne que j'ai proposée de cet ouvrage dans Francia-Recensio, revue de comptes rendus historiques de l'Institut historique allemand de Paris, 2010-11 (http://www.perspectivia.net/content/ publikationen/francia/

francia-recensio/2011-1/MA/toomaspoeg-peters-custot).

9. Martin 2001, p. 487-522, ici p. 504-505.

10. Ibid., p. 502

11. Ibid., p. 506. Sur la différence entre les comtés avant et après Roger II, voir Cuozzo 1989, en particulier p. 105-113.
12. C'est également ainsi qu'il est nommé dans un jugement daté de 1165, qui évoque la platea du comte Raoul (Trinchera $\left.1865, \mathrm{n}^{\circ} 167\right)$.

13. En 1165, les moines de S. Stefano del Bosco se plaignent en justice contre les capitaines des galères de Mileto qui, au service du roi, extorquent à des hommes donnés au monastère des services qui sont indus (Trinchera 1865, $\mathrm{n}^{\circ} 167$ ). Ces hommes de statut dépendant étaient dans la circonscription de Badolato et avaient été donnés au monastère 47 ans avant le document de 1165, soit en 1118, par un seigneur local, Carbouneris. Ils étaient inscrits sur la platea du comte Raoul, c'est-à-dire du père de Geoffroy, Raoul de Loritello. Ce dernier est mort avant 1111 : est-ce que cela signifie que Raoul était seigneur à Badolato tout en étant comte de Loritello, et avant la création du comté de Catanzaro? Cette situation est loin d'être improbable : on sait par ailleurs que Raoul, après avoir aidé le duc Roger Borsa dans sa lutte contre Adam, fils de Mihera Falluca, avait reçu en 1088 la moitié des possessions de ce dernier, devenant ainsi seigneur de Catanzaro (Martin 2001, p. 503). La seigneurie sur Badolato était donc dès l'origine rattachée à celle de Catanzaro. 
quelque peu "démembrée", le roi ait cru bon d'accroître encore l'aspect "éclaté» de son emprise territoriale en créant un autre comté, plus petit, qui pouvait couper en deux celui de Catanzaro : le comté de Squillace, attesté pour la première fois en $1154^{14}$. C'est là que l'analyse des actes de S. Stefano del Bosco, communauté monastique installée dans la région de Stilo, mais largement possessionnée dans celle de Squillace, et fortement liée au comté de Catanzaro, peut apporter des éléments complémentaires sur la politique des rois normands à l'égard des comtes calabrais. Il s'agit en particulier d'une série de trois diplômes royaux émis en faveur de S. Stefano del Bosco, qui s'enchaînent les uns aux autres et qui reviennent chacun successivement sur les concessions réalisées par le précédent document.

\section{S. STEFANO DEL BOSCO}

S. Stefano del Bosco est la branche cénobitique tardive d'un ermitage, celui de S. Maria de Turri, qui était lui-même la dernière fondation monastique de Bruno de Cologne ${ }^{15}$. Ce dernier, arraché par Urbain II aux solitudes de la Grande Chartreuse où il s'était installé depuis quelques années seulement, vint accompagner le pape dans le "Mezzogiorno" et s'installer, avec l'aide matérielle du comte Roger I ${ }^{\text {er }}$, dans les environs de Stilo, au diocèse de Squillace dans la Calabre méridionale : il y fonda l'ermitage de S. Maria de Turri où il mourut le 6 octobre 1101, soit quelques mois seulement après son bienfaiteur le Grand Comte, mort au mois de juin précédent. Une vingtaine d'années après la mort de Bruno, vers 1120, son second successeur, le magister Lambert, fonda un établissement cénobitique qui peu à peu prit l'avantage sur l'ermitage initial: S. Stefano del Bosco $^{16}$. Ce passage de l'ermitage collectif voulu par Bruno, à une forme assez traditionnelle de cénobitisme, sur un modèle franchement bénédictin, a été ensuite l'objet d'une construction historiographique largement élaborée par l'ordre cartusien après la canonisation tardive de Bruno au début du XVI ${ }^{\mathrm{e}}$ siècle, et dans laquelle la transition de l'érémitisme au monachisme collectif était considérée comme une "trahison" de la volonté de Bruno, consacrée par le passage de l'établissement à l'ordre cistercien, en 1193: dans cette reconstruction, l'entrée du monastère dans l'ordre cistercien fut considérée comme le transfert scandaleux de l'ordre cartusien à l'ordre cistercien, à l'instigation d'un homme indigne, le magister Guillaume de Messine ${ }^{17}$ dernier successeur de Bruno à la tête d'un établissement "cartusien" et premier prieur du même monastère, devenu abbaye cistercienne.

Jusqu'à il y a peu, l'historiographie ne s'était pas penchée sérieusement sur le passage calabrais de la vie de Bruno, ni sur les caractéristiques de son ultime implantation terrestre ${ }^{18}$ : il faut dire que les actes émis par Roger $I^{\text {er }}$ en faveur de Bruno, qui constituent quasiment les seules sources contemporaines, forment un dossier épineux du point de vue archivistique (les pièces ayant disparu en 1943 avec les fonds de l'Archivio di Stato de Naples, et bien des actes n'étant connus que par des éditions anciennes voire partisanes, comme celle de Benedetto Tromby ${ }^{19}$ ) comme du point de vue diplomatique: les faux, les actes interpolés, les copies côtoient les documents originaux, sincères ou authentiques, sans qu'il soit aisé de les distinguer, les canons de la diplomatique des comtes normands de Sicile n'ayant pas encore été fixés au moment de l'émission des privilèges de Roger I $^{\text {er }}$ à destination de Bruno et de ses compagnons. Par ailleurs, la grande majorité des documents comtaux pour la Calabre est alors rédigée
14. Trinchera 1865, $\mathrm{n}^{\circ} 150$.

15. La littérature entourant Bruno de Cologne est immense, et il n'est pas le lieu d'en déployer ici toute l'étendue, alors même qu'elle est souvent répétitive. On peut se référer à l'étude, un peu ancienne, mais détaillée, malheureusement assez peu diffusée, de Dom Maurice Laporte (Laporte 1960), ou consulter la biographie, plus accessible, de Bernard Bligny (Bligny 1984). Dom Jacques Dubois souligna avec vivacité la difficulté d'établir une vie de Bruno à partir de textes tardifs dont aucun ne mérite véritablement le nom de source (Dubois 1968, p. 27-54).
16. La date approximative de la fondation de S. Stefano del Bosco a été définitivement établie par Maurice Laporte (Laporte 1960, p. 292-304).

17. Le titre de magister, réservé aux supérieurs de S. Stefano del Bosco, est ce qui reste du souvenir de Bruno de Cologne, qui portait cette distinction probablement en raison de son passé d'écolâtre de Reims.

18. Font exception à la fois l'ouvrage fondateur de Dom Maurice Laporte, et, plus récemment, l'article d'Huguette Taviani-Carozzi (Taviani-Carozzi 2003, p. 59-79).

19. Storia critico-cronologica diplomatica 1775. 
en grec, l'usage de la langue grecque ayant prévalu jusqu'au règne de Roger II inclus : il n'y eut pas de chancellerie latine à Palerme avant le règne ducal de Roger II (1127) ${ }^{20}$. Tous ces éléments ont longtemps entretenu les équivoques nées de l'historiographie cartusienne sur Bruno, sa stature de fondateur, et la nature de la fondation calabraise ${ }^{21}$.

Toutefois, à l'époque de la formation et de l'évolution des comtés calabrais, c'est-à-dire après 1130 , les problèmes diplomatiques posés par ce fonds d'archives sont résolus par la fixation des règles de rédaction des diplômes royaux. Ceux d'entre eux qui sont émis pour la Calabre sont de moins en moins souvent rédigés en grec après le règne de Roger II, et de plus en plus souvent en latin ${ }^{22}$. Par ailleurs, on constate qu'après l'investissement important de Roger Ir aux côtés de Bruno, les souverains successifs ne favorisent plus autant l'ensemble des établissements nés de la fondation calabraise de Bruno (et parmi lesquels désormais S. Stefano del Bosco domine largement sur l'ermitage initial, S. Mari de Turri), et les actes royaux se font rares. Ainsi, à la mort quasi concomitante du Grand Comte (juin 1101) et de Bruno (octobre 1101), leurs successeurs respectifs, Roger II et le magister Lanvin, ont des relations plus distantes, non pas que la fondation soit négligée, mais parce que désormais, c'est à des personnalités plus locales

20. Ménager 1957, p. 7-30, ici p. 15 et 24 .

21. Je me permets de renvoyer au mémoire inédit que j'ai présenté pour mon Habilitation à diriger les recherches (soutenue le 3 décembre 2011 à l'Université de Paris 1), Bruno de Cologne en Calabre, et qui va être publié prochainement.

22. À partir du règne de Guillaume $\mathrm{I}^{\mathrm{er}}$, le renversement de tendance à la chancellerie est net : à cette époque, seulement $9 \%$ des actes recensés de la chancellerie royale sont en grec, sans compter quelques actes bilingues latin-grec, dont certains à destination de S. Stefano del Bosco. Le déclin se poursuit avec les successeurs de Guillaume I ${ }^{\mathrm{er}}$. La chancellerie de Frédéric II ne produit plus de privilèges en grec, même si le souverain conserve à ses côtés des notaires capables de rédiger des lettres en grec à destination de correspondants extérieurs, à commencer par son gendre Jean III Vatatzès.

23. On possède en particulier deux actes grecs du comte Geoffroy de Catanzaro, en 1131 et 1142, qui confirment le soutien de l'autorité publique au développement harmonieux de la seigneurie de S. Stefano del Bosco (Trinchera 1865, no 111 et Storia critico-cronologica diplomatica 1775, IV, Appendice, $\mathrm{n}^{\circ} 8$ p. 16 : Tromby publie le document en latin, mais précise qu'il s'agit d'un acte grec).

24. Rogerii II. 1987, n 67 p. 193-197, diplôme de confirmation des actes de S. Stefano del Bosco, 5 novembre 1144. Je pense pour ma part que ce document est une recomposition postérieure, voire tardive (du début du XIII ${ }^{\mathrm{e}}$ siècle) sur la base des archives de S. Stefano del Bosco, ce que j'expose qu'est dévolu le soin de protéger et d'enrichir l'établissement monastique : le comte de Catanzaro s'illustre en particulier dans cette fonction ${ }^{23}$, ce qui n'est probablement pas le signe d'une sorte de délégation locale de l'autorité souveraine en la matière. En effet, les institutions comme les individus n'obtiennent des diplômes et privilèges que s'ils en demandent : c'est donc que les moines et leurs abbés, désormais, dirigent leurs demandes vers les comtes et seigneurs locaux, plutôt que vers le grand comte, puis roi de Sicile. Ils considèrent donc probablement que les actes émis par les comtes et seigneurs locaux suffisent à garantir leurs biens et à les augmenter. On peut y voir la conséquence de l'éloignement physique du souverain, qui a transféré sa capitale de Mileto à Palerme, et qui est pour l'heure moins accessible, mais aussi le signe que les institutions monastiques calabraises ont tout à fait confiance dans les assurances que peuvent leur donner les actes des comtes de Catanzaro.

Toujours est-il qu'à l'époque de la royauté normande, S. Stefano del Bosco bénéficie de quelques préceptes royaux : un seul, fort douteux, pour la période royale de Roger II (1130$1154)^{24}$, deux documents de Guillaume I $^{\text {er25 }}$, deux de Guillaume $\mathrm{II}^{26}$ - sans compter une intéressante donation datée de 1168, et connue par un acte de définition des confins ${ }^{27}$ - et deux de Tan-

dans Bruno de Cologne en Calabre, cité note 21.

25. Guillelmi Regis Diplomata 1996, nº 3 p. 9-11, 20 décembre 1154 (= Trinchera 1865, $\mathrm{n}^{\circ} 153$ ) et ibid., $\mathrm{n}^{\circ} 88$ p. 88-90, mars 1166. Le premier privilège est un acte bilingue latin/grec, le second également, mais dont on n'a conservé que la partie latine.

26. Pour le règne de Guillaume II (1166-1189), l'analyse des diplômes royaux est délicate dans la mesure où on ne bénéficie pas d'une édition exhaustive des actes de ce souverain. Il faut se fonder sur les nombreux travaux de Horst Enzensberger. On y a repéré deux documents latins destinés à S. Stefano del Bosco : le premier de 1167 (Enzensberger 1981-1982, p. 23-61 $\mathrm{n}^{\circ} 6$ ) et le second de 1173, qui corrige le document émis en 1166 par Guillaume I ${ }^{\text {er }}$ (Enzensberger 1971, no 101 p. 127). Ce second document, qui nous intéresse le plus, a disparu, la seule édition disponible étant celle, fort imparfaite, de Benedetto Tromby (Storia criticocronologica diplomatica 1775, IV, Appendice I n ${ }^{\circ}$ 100, p. 117).

27. Il convient en effet d'ajouter aux deux diplômes de Guillaume II mentionnés ci-dessus une donation perdue datée de 1168 et concernant des biens fonciers, qui est attestée par un document publié par Benedetto Tromby (Storia criticocronologica diplomatica 1775, IV, Appendices, $\mathrm{n}^{\circ} 73$ p. 88). Tromby ne publie les documents qu'en latin, sans mentionner si l'original de départ était en latin ou en grec. Il se trouve que le document latin, qu'il dit avoir trouvé dans le cartulaire de S. Stefano del Bosco (daté des années 1200 et perdu) a un correspondant grec non daté, publié par Trin- 
crède $^{28}$. Ces diplômes royaux sont rédigés en latin, sauf ceux de Guillaume Ir , écrits en latin et grec. Il convient de les reprendre dans l'ordre chronologique, et on se penchera essentiellement sur les diplômes de 1166 (Guillaume Ir), 1173 (Guillaume II) et 1191 (Tancrède) qui intéressent indirectement l'histoire des comtés calabrais.

\section{LES ACTES ROYAUX DES DEUX GUILLAUME}

EN FAVEUR DE S. STEFANO DEL BOSCO, 1166-1173

Résidant, en mars 1166, dans son palais de Palerme, Guillaume I ${ }^{\text {er }}$ reçoit la visite de Landricus, magister du monastère Sancti Stephani de Bosco Harene (ce mode de dénomination, pour S. Stefano del Bosco, est unique, mais l'identification de l'établissement ne fait pas de doute $^{29}$ ). Le roi concède alors une redevance annuelle en nature au monastère, comptée en froment, orge, vin (pour célébrer les messes). Les céréales seront données annuellement par les baiuli de Stilo, le vin, par les baiuli de Squillace. De plus, le roi décide de confirmer la dotation annuelle de 500 tarins, somme que

de palatio nostro pro vestimentis et aliis necessariis hactenus recipere solebatis, de eodem palatio nostro singulis annis statuimus tribuendo.

Ces 500 tarins constituent donc un revenu établi avant 1166, revenu habituel, coutumier, dû par l'autorité publique. La concession de revenus publics à des seigneurs fonciers, notamment monastiques, de Calabre est extrêmement rare sous les souverains normands, en particulier le

chera 1865, Appendice II, nº 14, et qui était conservé à l'Archivio di Stato de Naples, donc issu non du cartulaire, mais du fonds d'archives de S. Stefano del Bosco. Voir PetersCustot 2008, II, p. 537-552.

28. L'édition des actes de Tancrède présente deux préceptes latins destinés à S. Stefano del Bosco : le premier date de mai 1191, et corrige le diplôme rédigé en 1173 par Guillaume II (Tancredi et Willelmi III 1982, $\mathrm{n}^{\circ} 12 \mathrm{p}$. 30-32) tandis que le second, du 5 février 1193, procède au transfert de l'établissement à l'ordre cistercien (ibid., no 31 p. 75-77) suite à un acte du pape Célestin III daté du 11 décembre 1192 (Kehr 1975, nº 21 p. 74).

29. D'une part, jusqu'au passage de S. Stefano del Bosco à l'ordre cistercien, seuls les supérieurs de cet établissement reçoivent le titre de magister, un héritage de Bruno, comme on l'a dit. D'autre part, certains des premiers diplômes tant comtaux que ducaux, émis en faveur de la fondation calabraise, de Bruno, du vivant de ce dernier, établissent que le premier d'entre eux, Roger II, on le sait ${ }^{30}$. On voit donc poindre ici, à la fin du règne de Guillaume ${ }^{\mathrm{er}}$, une lente évolution des pratiques royales envers les seigneuries monastiques, qui incluent désormais des concessions rarissimes avant lui. Toutefois, soulignons-le, il ne s'agit pas de la concession d'une parcelle d'autorité publique, mais de revenus publics.

En 1173, le roi Guillaume II reprend cette concession/confirmation pour la supprimer et la compenser par un privilège qu'il estime au moins équivalent : le diplôme royal, dont l'authenticité paraît indubitable ${ }^{31}$, supprime la concession de revenus publics réalisée en 1166, et lui substitue la donation du casale dit "de Conte" ${ }^{32}$. On peut donc momentanément conclure que le règne de Guillaume II mit en place les conditions d'une évolution radicale de la seigneurie de S. Stefano del Bosco. En effet, si les bienfaits que les deux rois Guillaume ont offerts aux moines de S. Stefano relevaient de la concession de revenus ou de droits publics (revenus annuels exigibles des baiuli locaux en 1166), les concessions de Guillaume II (un casale relevant du domaine royal - du moins le suppose-t-on - en 1173) ont transformé la seigneurie monastique dans une direction qui avait été proscrite auparavant par les souverains euxmêmes. Après la mort de Roger II et surtout à partir du dernier tiers du XII ${ }^{\text {e }}$ siècle, l'action royale envers la fondation de Bruno comprend désormais des privilèges qui vont largement au-delà des traditionnels dons d'hommes et de terres (on peut se demander si cette mutation n'est pas liée à l'épuisement du stock des terres à concéder, dans cette seconde moitié du XII ${ }^{e}$ siècle $\left.{ }^{33}\right)$. L'évolution

terrain concédé par Roger I ${ }^{\text {er }}$ était situé inter locum, qui dicitur Arena, et oppidum, quod appellatur Stilum : Becker à paraître, actes $\mathrm{n}^{\circ} 14$ et 31 et Ménager 1983, p. 3-35, p. 29. Le magister Landricus doit être identifié avec $\Lambda \alpha v \delta \rho v{ }^{\prime}$, qui en 1165 porte plainte au nom du monastère de $S$. Stefano del Bosco, contre les capitaines des galères de Mileto.

30. Martin 2008.

31. Schlichte 2005, p. 172 et n. 395.

32. Ce casale doit être distingué du castellum de Comite attesté dans un document de 1080, mais situé dans l'environnement directe de Salerne, au sud-est de la ville (Ménager 1980, no 34 p. 108-110).

33. On a constaté une croissance insigne des procédures judiciaires liées à des querelles de bornage et des contestations de terrain entre S. Stefano del Bosco et ses voisins dans la seconde moitié du XII ${ }^{\mathrm{e}}$ siècle et ultérieurement, reflet probable d'une pression foncière devenue difficile à rendre pacifique. Je renverrai là encore à Bruno de Cologne en Calabre. 
interne du monastère y est certainement pour quelque chose. Toutefois, si la création de S. Stefano del Bosco vers 1120 a déclenché, probablement, une mutation dans la nature même de l'établissement monastique et de sa stratégie économique, c'est grâce au roi que le monastère est prêt, dès les années 1160, à devenir une abbaye cistercienne; elle fonctionne du moins comme une abbaye bénédictine traditionnelle, et la question de la «trahison" des idéaux "cartusiens» de Bruno de Cologne ne se pose pas vraiment.

\section{TANCRÈDE, S. STEFANO DEL BOSCO ET LES COMTÉS CALABRAIS}

En 1191, Tancrède reprend à son tour la donation réalisée par Guillaume II en 1173, afin de la corriger $^{34}$ :

Hinc est quod, cum magnificentia nostra Casale Comitis, quod monasterium Sancti Stephani de Nemore dono regio possidebat, filiis Anfusi quondam comitis Squillacini, consanguinei nostri, pro eo, quod de comitatu Squilacii fuerat, restituissemus, eidem monasterio et fratribus eius in excambio competenti duximus providendum. Eapropter ipsi monasterio necnon tibi fratri Willelmo eiusdem monasterii priori tuisque successoribus et confratribus in perpetuum concedimus et donamus pro excambio predicti casalis ecclesiam Sancti Leontii cum casali suo et ecclesiam Sancti Fantini et ecclesiam Sancti Nicolai de Trivio cum omnibus iustis tenimentis et pertinentiis suis, quas Rainaldus de Busakano succentor cappelle nostre tenuit in prebenda a cappella castelli nostri Messane.

Depuis 1173, S. Stefano del Bosco possédait le casale de Conte, concédé pour compenser la perte contemporaine des revenus qui avaient été donnés par le roi en 1166; ce casale, on l'apprend en 1191, relevait du comté de Squillace, ce que le document royal de 1173 ne précisait pas. Tancrède restitue ce casale au comté de Squillace, et en l'occurrence aux fils d'Amfusus, son parent, qui avait été comte de Squillace ${ }^{35}$. Cette restitution atteste que la concession précédente du casale par Guillaume II lésait les droits légitimes du comte de Squillace, (d'où le silence, sans doute, que le diplôme de Tancrède maintient sur l'identité du roi par lequel la concession avait été réalisée). En échange et pour compenser la perte générée par cette récupération, Tancrède concède à $S$. Stefano del Bosco, qui semble devenir de plus en plus proche d'une abbaye cistercienne (le supérieur, déjà, n'est plus appelé magister mais prior) trois églises, Saint-Léontios, Saint-Phantin et SaintNicolas de Trivio, qui constituaient auparavant des prébendes de la chapelle du château royal de Messine, octroyées à un certain Rainaldus de Busakano, succentor de la chapelle.

Ce document invite à considérer de manière très détaillée l'histoire des comtés de la Calabre méridionale au XII ${ }^{e}$ siècle. On pourra y lire le rôle politique que les souverains faisaient jouer aux monastères - mais aussi aux évêchés - conçus comme des contre-pouvoirs non seulement économiques, mais aussi territoriaux : les enrichir pouvait aussi revenir à affaiblir l'emprise territoriale des grands, voire à les punir de leurs rebellions, toujours à craindre.

Squillace était une ville fortifiée importante, une des dernières places fortes de la résistance calabraise aux Normands ${ }^{36}$, placée ensuite sous l'autorité directe du comte Roger I ${ }^{\mathrm{er}}$, qui déplace et reconstruit la ville et crée un évêché latin en $1096^{37}$. Cette situation perdure au moins jusqu'en
34. Tancredi et Willelmi III 1982, n. 28, nº 12, Palerme, mai 1191, p. 30-32, ici p. 31 1. 5-14.

35. Amfusus est attesté comme tel en 1176 : Pirri 1733, I, p. 453-455. Il souscrit une charte en février 1177, en compagnie d'Hugues, comte de Catanzaro ( $M G H$ 1885, p. 94-95). Amfusus est mort avant août 1188, mais depuis peu, car à cette date sa femme Adélaïde émet une charte de donation en faveur de S. Stefano del Bosco afin d'exécuter la volonté que son mari avait émise de se faire enterrer, avec sa fille Marie, à l'église de S. Stefano, contre la concession d'une vigne dite de Marina et du moulin de S. Giacomo (Storia critico-cronologica diplomatica 1775, IV, Appendices, n XL p. 233 - la concession est confirmée dans un acte de Frédéric II, daté de mars 1224, mais jugé douteux par Huillard-Bréholles : Historia Diplomatica Friderici secundi 1852-1861, II, 2, p. 943-950). Adélaïde est vraisemblablement régente du comté à partir de 1188, mais un document grec rédigé en 1179 est daté, déjà, de la comtesse Adélaïde (Trinchera 1865 , $\mathrm{n}^{\circ}$ 195). Un document de l'impératrice Constance évoque encore, en 1196, la comtesse Adélaïde (Die Urkunden der Kaiserin Konstanze 1990, n 32). Guillaume, comte de Squillace et fils d'Amfusus, acte en 1201 en faveur de S. Stefano del Bosco (Trinchera 1865, $\mathrm{n}^{\circ} 251$ ).

36. Bougard-Noyé 1988, p. 215-230.

37. Regii Neapolitani Archivi 1861, p. 164-167, et désormais Becker à paraître, no 54 . 
$1145^{38}$. Puis est créé, soit à la fin du règne de Roger II, soit au tout début de celui de Guillaume $\mathrm{I}^{\mathrm{er}}$, un comté de Squillace, dont la première attestation se trouve dans un document grec, daté de $1154^{39}$. Il s'agit d'une donation à S. Stefano del Bosco, faite par Robert, fils de Robert de Terroun ${ }^{40}$, vassal du comte Evrard (Aberardos) de Squillace, pour l'âme des rois défunts, pour Guillaume $\mathrm{I}^{\mathrm{er}}$ et pour le comte Evrard. Jean-Marie Martin a montré d'une part que cet Evrard est un neveu de Robert Bovensis, le Robert de Boves, envoyé en exil par le roi de France pour trahison (et donc d'origine picarde) et qu'il est apparenté aux sires de Coucy; d'autre part que ce même Evrard devait être assimilé à celui qui, d'après Hugues Falcand, avait été accusé de trahison suite aux événements de 1160-1161 (il eut la langue coupée, et le comté de Squillace a dû alors passer dans d'autres mains) ${ }^{41}$. On peut donc placer dans la lignée de ces mesures répressives contre le comté de Squillace, la concession d'un casale appartenant à ce comté au monastère de S. Stefano del Bosco en 1173; ce qui permet, en passant, au roi de récupérer les revenus publics concédés au monastère en 1166. Le souverain fait d'une pierre deux coups

En 1176, le comte de Squillace est un certain Amfusus $^{42}$, dont il est plus que probable qu'il s'agisse de celui que Tancrède appelle "mon parent», on ne sait pas vraiment pour quelle raison, sinon qu'un des fils de Roger II portait ce nom $^{43}$. Cette parenté est crédible : confier des comtés à des parents du roi constituait une stratégie politique fermement établie ${ }^{44}$. En 1201, Guil-

38. Trinchera 1865, $\mathrm{n}^{\circ} 139,20$ mars 1145. Le roi Roger II confirme au nouvel évêque de Squillace Celsius les privilèges antérieurs, probablement dans le cadre de l'édit de révocation : voir Falkenhausen 1998a, p. 253-308, ici p. 306 n. 323.

39. Trinchera $1865, \mathrm{n}^{\circ} 150$.

40. D'après ce texte, Robert de Terroun est implanté à Cuculo, c'est-à-dire Monte Cùculo, à l'est de Polistena. Donc le comté de Squillace allait en 1154 au-delà du monastère «des ermites", au sud-ouest de la ville. Les comtés sont de toute façon absolument nouveaux dans la Calabre méridionale, donc un peu artificiels, sans forcément de continuité territoriale, ce qui pouvait être un façonnage conscient. Il convient de relever que, dans l'acte de création du nouveau diocèse de Squillace en 1096, Roger Ir donne à l'évêque le Castellum Cuculi.

41. Martin 2001, p. 504.

42. Il souscrit un acte royal en faveur de Monreale : Pirri 1733, p. 453-455.

43. Hugues Falcand 1897, p. 6, Amfusus est dit prince de Capoue. laume, fils du comte Amfusus, se dit "par la grâce de Dieu " comte de Squillace, usant d'une formule qui, abandonnée depuis la fondation du Regnum (1130), semble montrer que Guillaume a accaparé ce titre pendant la période anarchique de la minorité de Frédéric II. La région de Squillace connaissait donc bien l'affaiblissement de l'autorité royale pendant cette période ${ }^{45}$. Signe de la reprise en main royale, le comté de Squillace disparaît sous le règne effectif de Frédéric II, pour renaître à l'époque de Manfred.

Quand on considère la géographie de la région, la création vers 1154 du comté de Squillace, probablement peu étendu (vu que S. Stefano possédait déjà, au milieu du XII ${ }^{\mathrm{e}}$ siècle, une bonne partie de la région située au sud de Squillace ${ }^{46}$ ) mais inséré entre deux parties de celui de Catanzaro, peut être interprétée comme une mesure politique visant à faire contre-poids au seul comté de Calabre, un peu massif, celui de Catanzaro. En revanche, la cession, en 1173, du casale de Conte au monastère de S. Stefano, probablement dévoué au souverain, constitue une donation politique pour punir le comté de Squillace et réduire sa puissance, suite à la disgrâce d'Evrard. Lorsque le comté de Squillace passe, avant 1176, à Amfusus, parent du roi, au contraire, la situation devient gênante : ainsi, en 1191, dans les circonstances difficiles de la succession de Guillaume II, le roi Tancrède a pu préférer restituer au comté de Squillace un casale qui ne pouvait relever que de lui, quitte à compenser le manque à gagner pour le monastère, par la cession de trois églises dûment possessionnées.

44. Et illustrée notamment par tous les comtes mentionnés dans le Catalogue des Barons, et dont Errico Cuozzo a montré la parenté avec la famille du souverain : Cuozzo 1989, p. 115-120.

45. Trinchera 1865, $\mathrm{n}^{\circ}$ 251. La femme de Guillaume, Medina, "comtesse par la grâce de Dieu et du roi", souscrit en latin la donation de son mari à S. Stefano del Bosco. Dans un document de mai 1202, toutefois, Guillaume (qui suscrit et souscrit) et sa femme la comtesse, qui souscrit, le tout en grec, ne font pas état de cette "grâce de Dieu" (ibid., Appendice I, 9, p. 522). Il faut dire que la présentation de ce diplôme de donation permet à elle seule d'exalter les prétentions de Guillaume : l'acte prend la forme d'un sigillion grec (dont la forme diplomatique est cependant fortement altérée) dont les premiers mots sont en onciale : $\Sigma$ YГH $\Lambda \Lambda$ ION ГENAMENON ПАР ЕMOY ГОY $\Lambda$ ПЕ МОY KOMI $\Sigma$ KY $\Lambda$ $\triangle \mathrm{AKO} \Sigma \ldots$

46. En particulier les casalia de Olivadi, Montauro et Gasparina (voir Bruno de Cologne en Calabre, cartes de l'annexe 6). 
Quelles sont les concessions royales qui, en 1191, compensent la rétrocession du casale au comte de Squillace? Il s'agit de trois églises, SaintLéontios, Saint-Phantin et Saint-Nicolas de Trivio, toutes accompagnées de leurs biens et dépendances, et la première munie de son casale éponyme, dont on a mention ultérieurement ${ }^{47}$. Ces trois églises, dont deux apparaissent comme des biens de l'évêché de Squillace lors de sa redéfinition par Roger I ${ }^{\text {er }}$ en $1096^{48}$, constituaient avant 1191 des prébendes attachées à la chapelle du château royal de Messine, et étaient détenues comme telles par un certain Rainaldus de Busakano ${ }^{49}$. Si l'authenticité du document de Tancrède a pu poser question - il pourrait constituer le dernier d'une série de plusieurs faux, incluant le diplôme royal de Guillaume I ${ }^{\text {er }}$ de 1166, celui de Guillaume II de $1173^{50}$ - un document grec vient confirmer, sinon l'authenticité, du moins la vraisemblance de son contenu. En effet, en 1184, le chapelain de SaintLéontios, un certain Rainaldos, est l'auteur d'un document de permutation de terre avec un certain Philippe $^{51}$. Il est plus que probable que ce Rainaldos soit le Rainaldus de Busakano mentionné par l'acte de Tancrède. Que Saint-Léontios, Saint-Nicolas de Trivio et Saint-Phantin soient des prébendes d'un chapelain du roi remonte au moins au règne de Guillaume II. Du reste, si le document de 1184 s'est retrouvé dans l'Archivio di Stato de Naples, comme l'indique Francesco Trinchera, c'est probablement parce qu'il relevait des archives de S. Stefano del Bosco. Il avait dû être inséré par les moines dans leurs archives au moment de la donation de Saint-Léontios en 1191.

\section{S. STEFANO DEL BOSCO, SAINT-LÉONTIOS ET LES COMTÉS CALABRAIS}

Lorsque le petit monastère de Saint-Léontios fut confié en 1191 à S. Stefano del Bosco, toutes les archives de Saint-Léontios y furent déposées. C'est ainsi qu'on a pour ce monastère de fondation byzantine une continuité de documentation, qui tranche avec l'ampleur somme toute modeste de l'établissement, et qui permet de reconstituer les phases de son destin ${ }^{52}$. L'histoire houleuse des comtés de Catanzaro et de Squillace, dans la seconde moitié du XII ${ }^{e}$ siècle, peut permettre de comprendre ou de dater certains de ses documents d'archives, conservés seulement dans d'anciennes éditions qui ne satisfont plus aux critères scientifiques actuels, mais aussi de comprendre les évolutions des dominations territoriales.

Saint-Léontios est un monastère calabrais de fondation byzantine : vers 1050, il est enregistré dans le brébion de la métropole de Reggio ${ }^{53}$ et dès 1044, il fait l'objet d'un jugement à son avantage dans une querelle foncière ${ }^{54}$. En 1096, il est mentionné dans la liste des biens que Roger I ${ }^{\text {er }}$ a établie pour l'évêque de Squillace ${ }^{55}$, ce qui signifie que le comte Roger Ir a accaparé dès la fin de la conquête de la Calabre méridionale (1059-1060, la dernière poche de résistance étant précisément Squillace) un grand nombre de biens qui rele-
47. Trinchera 1865, no 324, 1271 : un des auteurs, Nicolas de Bona, est habitant du chôrion de Saint-Léontios.

48. Références supra en n. 37.

49. ...quas Rainaldus de Busakano succentor cappelle nostre tenuit in prebenda a cappella castelli nostri Messane.

50. C'est l'hypothèse que fait Herbert Zielinski, l'éditeur des diplômes de Tancrède et de Guillaume III, pour l'écarter ensuite : Tancredi et Willelmi III 1982, p. 30-31.

51. Trinchera $1865, \mathrm{n}^{\circ} 220$.

52. Ce que j'ai partiellement fait dans Peters-Custot 2008.

53. Saint-Léontios est en effet non seulement mentionné dans le brébion de la métropole byzantine de Reggio, rédigé autour de 1050 pour répertorier les redevances dues à la métropole byzantine de Reggio de Calabre, mais il y bénéficie même de deux périorismoi différents : Brébion 1974, l. 133 et 146 (texte grec), et p. 32-33 (traduction française).

54. On dispose d'un jugement - perdu en 1943, mais publié par Francesco Trinchera - rédigé en novembre d'une indiction 13, par Stéphane, ek prosôpou du stratège de Calabre Antiochos, suite à une plainte déposée par Cosmas, higoumène de Saint-Léontios de Stilo contre les fils du protospathaire
Synator, qui se seraient emparés illégalement d'un moulin du monastère situé sur le fleuve de Stilo, au lieu-dit Panarin (Trinchera 1865, $\mathrm{n}^{\circ} 44$ ). Francesco Trinchera date, à tort, cette sentence de 1059, alors que la date de 1044 convient nettement mieux à l'environnement exclusivement byzantin dont témoigne la sentence, comme l'a indiqué André Guillou dans son édition du brébion de Reggio; toutefois, André Guillou revient sur la date de 1059 dans l'édition des actes de Saint-Jean-Théristès, car il estime que le jugement de Stéphane, qui utilise le périorismos de Saint-Léontios est postérieur au brébion, et que le brébion ne peut dater que de 1050 "environ": Saint-Jean-Théristès 1980, p. 277. Le brébion de Reggio étant toutefois constamment mis à jour, on peut supposer que la version utilisée par Stéphane est une version antérieure à celle qu'André Guillou a éditée. La description des limites de Saint-Léontios a d'ailleurs pu être retouchée entre les deux versions. On peut aussi admettre que la version du brébion éditée par A. Guillou était antérieure à 1044. Je traite de l'ensemble de ce dossier dans Peters-Custot 2008.

55. Références supra en n. 37. 
vaient à l'époque byzantine de l'Église de Reggio, pour pouvoir, soit les garder en son contrôle, soit les concéder à ses fidèles ${ }^{56}$. En 1096 donc, SaintLéontios est propriété de l'évêque de Squillace. Lorsque, le 20 mars 1145, Roger II émet une charte grecque de confirmation de certains biens de l'évêque de Squillace ${ }^{57}$, Saint-Léontios n'y apparaît pas, mais ce n'est pas un argument absolument déterminant, car le roi ne confirme dans ce document que les noms des hommes donnés en 1096, et non les biens fonciers ou les églises, à l'exception d'une petite donation effectuée par sa mère Adélaïde lors de la période de régence de cette dernière (entre 1101 et $1112^{58}$ ). En 1168, Saint-Léontios apparaît dans la description des confins (périorismos) de biens concédés par le roi Guillaume II et sa mère à S. Stefano del Bosco : il semble alors indépendant de ce dernier ${ }^{59}$. En 1184, il est aux mains du chapelain Raynaldus ${ }^{60}$, ce qui signifie que le monastère était retourné déjà à cette date dans le giron royal. En 1191 enfin, Tancrède donne Saint-Léontios à $\mathrm{S}$. Stefano del Bosco.

Visiblement, Saint-Léontios a des archives bien tenues, qui sont transférées en 1191 à S. Stefano del Bosco $^{61}$, et où sont conservés trois documents essentiels, dont certains sont issus ou copiés de l'époque byzantine, même si aucun d'entre eux

56. On a pu constater un autre de ces prélèvements sur les anciens biens de la métropole byzantine de Reggio lors de la dotation de l'ermitage de Bruno de Cologne, S. Maria de Turri, qui reçoit de Roger I ${ }^{\mathrm{er}}$, en 1094, une église byzantine mentionnée dans le brébion de la métropole byzantine de Reggio, S. Maria d'Arsafia (voir Bruno de Cologne en Calabre). Ces deux concessions issues du patrimoine métropolitain de Reggio datent du début des années 1090, et suggèrent une captation antérieure d'une partie au moins de ce patrimoine par Roger I ${ }^{\text {er }}$ au détriment d'un siège métropolitain qui, précisons-le, est depuis des années à la disposition d'un prélat latin, ce qui permet de récuser toute interprétation de ces prélèvements comme manœuvre de "latinisation" d'un diocèse fortement marqué par la présence hellénophone.

57. Trinchera $1865, \mathrm{n}^{\circ} 139$.

58. Voir Falkenhausen 1998b, p. 87-115: l'annexe de cette étude présente les regestes des actes d'Adélaïde, p. 105-115. On se reportera au numéro 14, où l'auteur identifie le privilège d'Adélaïde confirmé par Roger II en 1145. Adélaïde avait offert, en 1109 ou 1110 le monastère de S. Maria de Roccella, alors dirigé par Gérasimos, à l'évêque de Squillace, avec tous ses biens. Gérasimos est un nom très répandu chez les moines et higoumènes italo-grecs de l'époque. Cet établissement semble donc être un des monastères grecs de la région de Squillace ou de ses environs : d'après Ermanno Arslan, cet établissement peut être identifié avec un ancien oratoire, qui porte le titre de S. Maria della Roccella, mais qui est distinct du grand édifice en ruine proche, mais situé n'est daté : outre deux listes d'hommes de SaintLéontios, appelées katonoma, et qu'on reprendra plus $\operatorname{tard}^{62}$, on y trouve en particulier un document grec détaillant le périorismos de SaintLéontios, document reprenant largement la partie du Brébion de la métropole de Reggio qui attestait des limites du monastère de Saint-Léontios à l'époque byzantine ${ }^{63}$, ainsi qu'au jugement d'une querelle de bornage en $1044^{64}$.

La question de la datation de ces trois documents, les deux katonoma et le périorismos, est importante car elle est liée aux modifications de la géographie administrative de la Calabre. En effet, on sait que les inventaires de biens monastiques sont souvent dressés au moment où un monastère change de statut, de seigneur ${ }^{65}$, ce qui engage, pour ces trois documents de Saint-Léontios, une foule de possibilités, en raison des divers remaniements de la carte des comtés calabrais dans la seconde moitié du XII e siècle, mais aussi du fait que ces actes n'ont probablement pas la même datation. Une interprétation a été avancée, qui apparente ces textes à des annexes rédigées en 1144, dans la suite d'un document royal de confirmation des biens de S. Stefano del Bosco, ce qui est sans doute contestable, et on ne la retiendra pas ici $^{66}$.

L'acte de description des confins de Saint-

plus à l'ouest, portant le même titre, et appelé également "La Roccelletta" : cette seconde église est plus tardive, et la date de sa construction oscille entre la fin du $\mathrm{XI}^{e}$ et le milieu du XII ${ }^{e}$ siècle, peut-être entre 1130 et 1150 (Arslan 1988, p. 205-206).

59. Saint-Léontios apparaît dans les confins d'une liste grecque de bien concédés par le puissant roi (anonyme) à S. Stefano del Bosco (Trinchera 1865, Appendice II, nº 14) et que j'ai datée, par recoupement avec un document édité en latin par Tromby, de 1168 (Peters-Custot 2008).

60. Trinchera 1865, $\mathrm{n}^{\circ} 220$.

61. Et de là à l'Archivio di Stato de Naples, où tous ces documents furent perdus en 1943.

62. Trinchera 1865, Appendice II, $\mathrm{n}^{\circ} 16$ et 17 .

63. Trinchera 1865, Appendice II, nº 15 et Brébion 1974, l. 133 et 146.

64. Trinchera $1865, \mathrm{n}^{\circ} 44$.

65. On se reportera à l'acte par lequel le seigneur de Briatico, Guillaume Capriol, dresse l'inventaire des biens d'un petit monastère grec de fondation byzantine et semble lui rendre ces biens, deux années seulement après l'aboutissement de la conquête normande de la Calabre méridionale, en 1062 : Rognoni 2004, $\mathrm{n}^{\circ} 11$. Il est probable que ce seigneur, après avoir reçu une terre du comte Roger I ${ }^{\text {er }}$, comprenant ce monastère, le «redonne» à son ancien propriétaire, auquel il s'est substitué en droit, l'higoumène.

66. Voir Johns 2002, p. 117 n. 11 : évoquant les diplômes de confirmation des privilèges émis par Roger II dans le cadre 
Léontios, composé sur la base d'un document de l'époque byzantine, date probablement soit de la fin de l'époque byzantine, soit des débuts de l'époque normande, mais on ne saurait être plus précis, même si les révisions apportées sont de détail ${ }^{67}$, et suggèrent une rédaction courant du $\mathrm{XI}^{\mathrm{e}}$ siècle, peut-être encore à l'époque byzantine, et en tout cas au plus tard à l'occasion de la concession de Saint-Léontios à l'évêque de Squillace en 1096. La conservation de cet acte après la donation de Saint-Léontios à S. Stefano del Bosco laisse songeur. Peut-on penser qu'en conservant en 1191 des documents byzantins, le monastère de S. Stefano del Bosco considérait les confins d'un monastère établi près $d^{\prime} u n$ siècle, voire un siècle et demi plus tôt, comme toujours valables? Invoquer le rôle symbolique du passé ne suffit peutêtre pas pour expliquer ce conservatisme archivistique d'une abbaye fortement marquée par le pragmatisme économique. Toutefois, la valeur perpétuelle de l'écrit, même s'il a été émis lors d'une domination périmée depuis plus de cent ans, permettrait sans doute au monastère de S. Stefano del Bosco de revendiquer des terres définies pour Saint-Léontios dans le Brébion byzantin de Reggio. Le remploi de documents byzantins suggère un usage ininterrompu, à l'époque normande, de certains documents de gestion territoriale et économique datés de l'époque byzantine. Les confins de territoires, du moins, sont relativement stables, l'inertie de la toponymie étant pratiquement sans limite (mais pas sans piège).

En revanche, toute opération de recensement nominal de la population a tendance à devenir rapidement désuète. C'est pourquoi la collecte, la conservation, la copie de ces inventaires d'hommes spécifiques à la Calabre et à la Sicile normandes qu'on appelle plateae, (en arabe, djarâ'id, en grec, katonoma) ${ }^{68}$, voire la fabrication de faux sur la base d'anciennes listes de dépendants ${ }^{69}$, ne laissent pas d'étonner. Elles doivent astreindre l'historien à reconsidérer avec un regard aigu ces documents, symboliques, mais régulièrement de l'édit de révocation, et actés pour la Calabre et la Sicile entre octobre 1144 et mars 1145, l'auteur considère que la seconde platea non datée de Saint-Léontios (Trinchera 1865, Appendice II, $\mathrm{n}^{\circ}$ 17) est à placer dans la catégorie des documents de confirmation. L'auteur se réfère à Vera von Falkenhausen (Falkenhausen 1998, p. 256 et n. 10). Or, cette dernière, en effet, pour illustrer la constatation fort juste (et très éclairante sur le maniement politique de la diversité linguistique) que, dans le cas des actes souverains émis en Calabre en latin, les données pratiques (confins des terres, noms des vilains donnés) apparaissaient en grec dans un document annexe, donne pour exemples les quatre documents non datés placés dans le second appendice de l'édition de Francesco Trinchera (Trinchera 1865, Appendice II, 14, $15,16,17)$ qu'elle assimile à des annexes en grec d'une donation latine de Roger II, datée du 5 novembre 1144 (Rogerii II. 1987, nº 67). De nombreux éléments contredisent cette interprétation : d'une part, le diplôme royal de 1144 n'est pas une concession de biens mais une confirmation d'actes grecs et latins antérieurs, nombreux et autonomes (toutefois, les souverains profitent souvent de confirmations pour ajouter des concessions nouvelles, qu'il est parfois difficile de distinguer des confirmations de concessions anciennes). Signalons toutefois que ce document royal de 1144 ne mentionne à aucun moment l'église de Saint-Léontios, et les documents confirmés dans cet acte correspondent à des donations pour la plupart dûment identifiées (voir Peters-Custot, Bruno de Cologne en Calabre, cité n. 21, Annexe 1). Surtout, ces quatre pièces d'archives assemblées artificiellement par l'édition de Trinchera ne composaient en fait certainement pas un groupe cohérent, ni les quatre annexes d'une même donation: le premier périorismos (Trinchera 1865, Appendice II, 14), correspond à un acte de délimitation de confins faisant suite à une donation du roi Guil- laume II en faveur de S. Stefano del Bosco, datée de 1168 (voir Peters-Custot 2008). Le second périorismos correspond à une copie mise à jour du kodex d'époque byzantine, c'est-àdire de la partie du registre cadastral byzantin correspondant au monastère de Saint-Léontios et à ses biens (Peters-Custot 2008 et Falkenhausen 2012, p. 543 n. 63). Pour ce qui est des deux listes d'hommes (Trinchera 1865, Appendice II, 16 et 17), si la date de 1144 peut être cohérente pour le premier katonoma édité (le $\left.\mathrm{n} .^{\circ} 16\right)$, en revanche, le second $\left(\mathrm{n}^{\circ} 17\right)$ est plus tardif, comme on le verra. Enfin, point déterminant, le monastère de Saint-Léontios et ses dépendances n'ont pas été concédés à S. Stefano del Bosco par Roger II, mais bien plus tard, en 1191, par Tancrède.

67. Voir l'analyse du périorismos de Saint-Léontios (Trinchera 1865, Appendice II, $\mathrm{n}^{\circ} 15$ ) qui passa des archives de SaintLéontios à celles de S. Stefano del Bosco en 1191, dans Peters-Custot 2008, p. 539-542. Le periorismos non daté de Saint-Léontios contient en effet de petites variations par rapport au périorismos de Saint-Léontios dans le brébion de Reggio, qui peuvent laisser penser qu'il lui est légèrement postérieur, et peut-être des débuts de l'époque normande, attestation du remploi par les Normands des documents fonciers calabrais de la période byzantine.

68. Sur les djarâ'id, je renvoie aux travaux de Jeremy Johns (Johns 2002) ainsi qu'à ceux d'Annliese Nef, et notamment à Nef 2000, p. 579-607 et Nef 2011, p. 481-566; sur les plateae, outre les contributions qui figurent dans le volume attenant à la publication de la platea de Cosenza (début du XIII ${ }^{e}$ siècle) : Cuozzo - Martin 2009. Je me permets de citer Peters-Custot 2008, Peters-Custot 2012a et 2012b.

69. Comme on le constate à plusieurs reprises dans le fonds des actes de S. Stefano del Bosco : voir Bruno de Cologne en Calabre. 
ajournés, ce qui suggère un usage pratique permanent qui justifie ces ajournements. Les listes d'hommes et leurs ajournements sont forcément dépendants d'un événement relativement proche pour que cela ait un sens.

Les deux katonoma des hommes de SaintLéontios, qu'on appellera ici "katonoma 16" et «katonoma 17», d'après l'édition de Francesco Trinchera ${ }^{70}$, sont également difficiles à dater. L'un de ces textes (le 16) évoque le roi ainsi que le comte Geoffroy (de Catanzaro, mort vers 1145), on a donc pensé qu'il datait des années 1132-114571. Toutefois, certains des individus mentionnés dans ces listes apparaissent nettement comme souscripteurs de documents des années 1155-1164 plutôt que $1130-1140^{72}$. Ce qui est quasiment certain, c'est que les deux katonoma ne sont pas émis à la même période, du moins c'est ce qu'on peut légitimement soupçonner d'après leur composition, et d'après le fait qu'aucun nom n'est commun entre les deux listes. Le document numéroté 16 est moins bien organisé, il mentionne des hommes sans ordre ni précision de statut et paraît relever d'une situation encore transitoire : certains individus recensés comme "hommes» de SaintLéontios dans cette source sont dits "hommes" d'un autre seigneur, à qui ils paient encore certaines redevances : Basile Kanakos, "homme» de Robert Argoukos, paie le dôma à ce dernier. Arkadios Korépsogénès est l'homme de Richard Bilaros et paie à ce dernier le dôma. Nicolas de Bordonaro est l'homme du comte Geoffroy (de Catanzaro) et lui paie le dôma... En revanche, le document numéroté 17 dans l'édition de Trinchera, tout en se limitant à un relevé des noms d'hommes, sans précision de redevances, les présente de manière ordonnée, en introduisant des catégories (ces précisions s'insèrent lentement dans la documenta-

70. Trinchera 1865, Appendice II, 16 et 17.

71. C'est la datation que j'avais proposée dans Peters-Custot 2008.

72. Trinchera 1865, Appendice II, 16 et 17 : Pierre Mélachrinos, mentionné dans le katonoma $\mathrm{n}^{\circ} 17$, souscrit un acte daté de 1164 (ibid., n ${ }^{\circ}$ 165) avec un certain Calocyre Francopetros, qui n'est pas sans rappeler le Frankopetros de la katonoma $\mathrm{n}^{\circ} 16$. Calocyre Francopetros achète des champs dans la région le 13 février 1155 (Saint-Jean-Théristès 1980, n² 24). La dernière souscription de ce document de 1155 est celle d'Oursos Phoberos; or, Arkadios Phoberos est un des hommes recensés dans le katonoma $\mathrm{n}^{\circ} 16$ - signalons $\mathrm{qu}^{\prime}$ Arkadios est la version grecque du latin Ursus, Ours.

73. Carocci 2009, p. 205-241, ici p. 227 sq., et Peters-Custot tion calabraise à partir de la seconde moitié du $\mathrm{XII}^{\mathrm{e}}$ siècle ${ }^{73}$ ) : d'abord les "hommes" (anthrôpoi) de Saint-Léontios, puis les akkoumendamenoi, enfin les «vilains" de Saint-Léontios, une liste qui compte de nombreux noms en commun avec les «hommes» (anthrôpoi) de Saint-Léontios ${ }^{74}$. Il est probable que la deuxième partie du document soit une mise à jour de la première : les noms personnels associés à certains cognomina récurrents changent d'un bout à l'autre de la liste, ce qui signifie qu'un descendant a repris la place d'un aïeul ou d'un quelconque parent ${ }^{75}$. Si le document 16 parle du roi et du comte Geoffroy de Catanzaro, et correspondrait donc plutôt à la période 11321145 , et plutôt vers 1150 , le document 17 mentionne des hommes attestés plutôt vers 1160-1165.

Il convient maintenant de rapprocher les faits onomastiques relevés dans les katonoma 16 et 17, des modifications administratives contemporaines, qui pourraient légitimer la rédaction de ces listes par un transfert de propriété sur Saint-Léontios. En 1096, ce monastère relève de l'évêque de Squillace. Le katonoma 16 semble dater des années 1150 : on ne saurait donc en rapprocher la rédaction de la création, vers 1132, du comté de Catanzaro, lequel d'ailleurs provient de la transformation en comté de la seigneurie déjà constituée de Catanzaro. Il est plus tentant de le rapporter à la création du comté de Squillace, vers 1154 , ce qui implique que le monastère de SaintLéontios a été retiré des biens du diocèse de Squillace et est retourné dans le giron du souverain entre 1096 et 1154 (mais on n'a guère d'idée pour affiner ces dates); vers 1154, le roi aurait donné le monastère de Saint-Léontios au comte de Squillace, à l'occasion de la fondation de ce comté, créé pour amoindrir celui de Catanzaro, ce qui explique du reste que certains des hommes de

2012a.

74. Voici quelques exemples de noms présents dans les deux parties du texte: Pierre Mélachrinos (voir aussi Trinchera $\left.1865, \mathrm{n}^{\circ} 165,1164\right)$, Consta de Kataro (puis de Katarès) mentionné trois fois, Oursos Géracitanos (de Gerace), Basile Gitzos, André Tourkos... les termes d' "homme" et de "vilain" sont donc équivalents. Sur ce sujet, voir aussi Patlagean 2008, II, p. 529-536.

75. Arkadios Roupharès puis Jean Roumpharès, Philippe, puis Nicolas, puis Basile Demennitès, Arkadios puis Nicolas Langobardos, Pierre puis Jean Rousios (on relève un Nicolas Rousios dans le katonoma 16), Barounès puis Nicolas Baronos, les enfants de Xanthis, puis Théodore de Xanthis... 
Saint-Léontios doivent encore des redevances au comte Geoffroy.

La suite des événements permet de proposer une datation pour le second katonoma, le 17. En effet, si en 1154 Saint-Léontios est donné au comte de Squillace, il a dû lui être retiré avant 1191, quand Tancrède donne le monastère à $S$. Stefano del Bosco. Or, en 1160-1161, le comté de Squillace change de main (ou reste temporairement vacant) suite à la déchéance d'Evrard : il est possible que le roi ait voulu, parallèlement, amoindrir le patrimoine et la puissance foncière du comté de Squillace, afin d'éviter que d'autres velléités de rébellion ne soient rendues possibles; $c^{\prime}$ est donc à ce moment que le monastère de Saint-Léontios aurait pu revenir dans le domaine royal, avant d'être concédé en 1191 : de là la rédaction du second katonoma.

Dès lors, on peut proposer de considérer que le premier katonoma a été réalisé au moment de la fondation du comté de Squillace (en 1154, c'est-àdire après la mort de Geoffroy de Catanzaro, mais l'inertie de ce genre de document est sans doute une explication raisonnable de la mention du comte), en prenant le monastère sur les biens de l'évêque de Squillace, et le second, vers 1161-1170, au moment où le roi prélève des biens du comté de Squillace pour les confier, d'abord à son chapelain, avant 1184, puis à S. Stefano del Bosco.

\section{CONCLUSION}

Il paraît nécessaire de ressaisir les données qu'on a pensé établir, en raison de la complexité des faits qui croisent les rois, les comtes calabrais, les monastères, les évêchés, ou plutôt qui montrent un jeu dans lequel les monastères, les comtés et les évêchés sont autant de cartes que le roi joue successivement pour maintenir les fragiles équilibres nécessaires à la stabilité de sa domination dans la Calabre méridionale.

À partir du moment où la domination normande paraît avoir vaincu les dernières résistances dans la Calabre (à Squillace, dans les années 10591060), il est probable que Roger I $^{\text {er }}$ accapara largement les terres disponibles, en particulier celles des métropoles et évêchés byzantins, afin d'en redistribuer une partie à ses fidèles (par exemple, le sire de Briatico avant $1062^{76}$ ), et de garder directement la main sur des ensembles vastes. $\mathrm{Au}$ début des années 1090, on le voit distribuer des églises issues du patrimoine de la métropole byzantine de Reggio à des institutions ecclésiastiques et monastiques qu'il contrôle étroitement (la fondation de Bruno de Cologne, qui reçoit S. Maria d'Arsafia, ou l'évêché qu'il reconstruit à Squillace, et qui reçoit Saint-Léontios).

Peu après la fondation de la royauté, son fils et successeur Roger II transforme la seigneurie de Catanzaro en un comté, vers 1132. Seul comté de la Calabre méridionale, celui-ci, aux mains de la famille de Loritello, reprend localement les missions de protection des monastères qui avaient été celles du comte de Sicile avant 1130, et prospère peut-être un peu trop. Le roi (Roger II ou Guillaume Ir) autour de 1154, le scinde en deux, créant le comté de Squillace, toutefois sans véritablement amputer le comté de Catanzaro : on peut en effet faire l'hypothèse que le roi reprit Saint-Léontios à l'évêché de Squillace, et le confia au comte de Squillace, équilibrant les forces de la région.

Un premier tournant advient vers 1160-1161, lorsque le comte de Squillace Evrard se soulève. Le souverain perçoit la menace et, après avoir brisé la révolte, renforce l'institution monastique qui est en passe de devenir un des grands seigneurs fonciers de la zone, S. Stefano del Bosco : le roi prélève en 1166 une partie des revenus publics de Squillace au bénéfice de ce monastère, concède en 1168 des biens transmis par le biais du stratigot de Squillace, puis change de méthode en 1173, et récupère les revenus publics donnés en 1166 tout en arrachant au comté de Squillace, peut-être encore sous tension, un casale dit "de Conte», là encore pour S. Stefano del Bosco. Par ailleurs, il est probable que dans ces années-là, vers 11611170 , et en tout cas avant 1184, Saint-Léontios change encore de mains, et, détaché du comté de Squillace, retourne dans le giron du roi, qui en fait une prébende du chapelain de Messine.

Deuxième retournement, celui-ci en faveur du comté de Squillace : en 1176, voire peu avant, il est confié à un "parent" du roi, Amfusus. Vu la nouvelle donne, il n'est plus question de le maintenir dans une situation basse, le casale de Conte 
est donc repris à S. Stefano del Bosco, en 1191, et redonné au comte de Squillace, tandis que le monastère de $S$. Stefano del Bosco récupère, en guise de compensation, des établissements qui étaient des prébendes du chapelain royal : en particulier, Saint-Léontios. Derrière ce nom d'exéglise byzantine se trouve très certainement une cellule de production agricole, voire un village, en tout cas un ensemble d'hommes désormais dépendants de S. Stefano del Bosco.

Ainsi, la stratégie royale de contrôle politique de la Calabre méridionale s'appuie sur les monastères et les évêchés, et les concessions qui paraissent à la limite de l'illégalité et de l'irrespect de l'autorité publique, comme celle de 1173, sont en fait des moyens de limiter le pouvoir concurrentiel des comtes et des vassaux importants. Les monastères, que ce soit $S$. Stefano del Bosco ou une ancienne fondation byzantine comme SaintLéontios, sont surtout appréhendés par le pouvoir royal, dans ce contexte, comme des entités territoriales et économiques avec lesquelles on peut jouer pour diviser, amoindrir, punir les comtes et les seigneurs concurrents.
Mais on peut aller plus loin que cette constatation, avouons-le, peu originale. Il est très clair en effet que toutes ces manœuvres servent autant à affirmer la prééminence du souverain qu'elles en manifestent la puissance : les rois déstabilisent les contenus des comtés, des monastères et des évêchés tant qu'ils en ont les moyens, et pour montrer précisément qu'ils en ont les moyens, et que les cartes sont en leurs mains. Leur retour permanent sur les concessions précédentes n'est pas un aveu d'indécision ou de faiblesse, au contraire : la précarité (relative) que créent ces revirements renforce la position d'un souverain qui surplombe les autres acteurs du jeu régional, l'instabilité des acteurs mineurs faisant la stabilité de l'acteur majeur. La minorité de Frédéric II signe donc logiquement l'arrêt brutal des ces manipulations incessantes, tandis que des comtés autoproclamés foisonnent de manière un peu anarchique sous l'effet de l'affaiblissement temporaire du pouvoir royal.

On aperçoit donc surtout, à travers quelques documents de fonds monastiques calabrais, le jeu déséquilibré qui permet au roi de Sicile de garder la main sur les terres calabraises grâce à la préser-

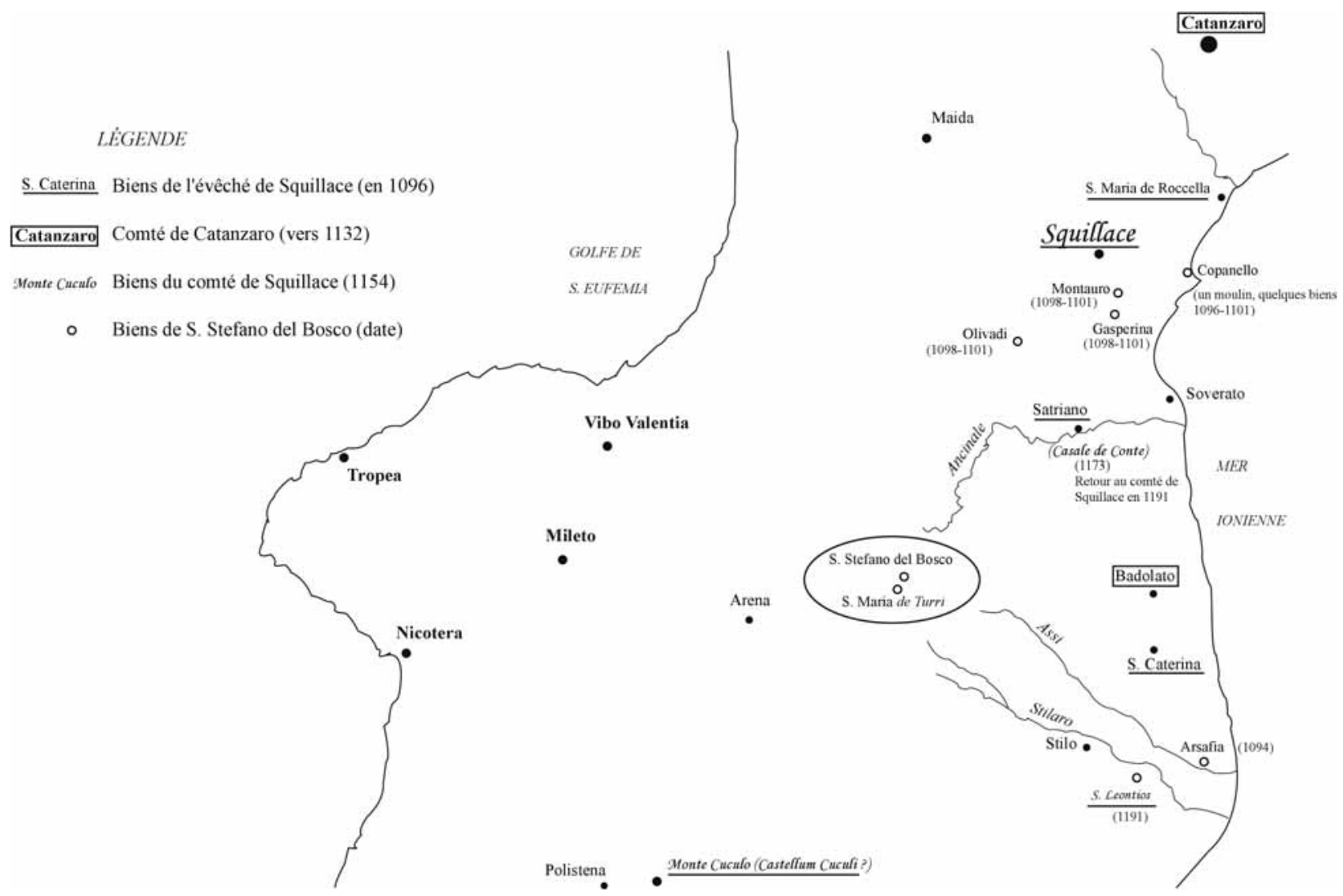

La région de Squillace, entre évêché, comtés et S. Stefano del Bosco. 
vation de droits régaliens dont il monopolise la détention (en particulier les revenus publics) mais aussi grâce à sa position de chef de l'Église : jeu impérial s'il en est, où les partenaires du roi, même s'ils sont soutenus, ménagés et entendus dans leurs demandes, ne sont pas en situation de force : évêchés, monastères et comtés doivent beaucoup au souverain.

Il apparaît très clairement que les rois normands, dès les années 1150, considèrent $S$. Stefano del Bosco comme une abbaye classique, avec ses moyens, ses prétentions à l'expansion et à la puissance foncière et économique, son instrumentalisation possible pour l'ancrage territorial de la domination souveraine. Dès avant le passage à la tutelle cistercienne, le supérieur est nommé prior, et non plus magister, et plus rien ne rattache ce monastère à la petite fondation érémitique de Bruno de Cologne. L'appellation "des ermites", qui avait longtemps résisté, accolée au nom de

\section{Sources et bibliographie}

Arslan 1988 = E. A. Arslan, La Roccelletta, dans R. Spada (éd.), Da Skylletion a Scolacium. Il parco archeologico della Roccelletta. Mostra di Milano, maggio 1988, RomeReggio di Calabria, 1988, p. 205-210.

Becker à paraître $=$ J. Becker, Edizione critica dei documenti greci e latini del conte Ruggero I di Calabria e di Sicilia (1080-1101), Rome, à paraître (Ricerche dell'Istituto storico germanico di Roma, 9).

Bligny 1984 = B. Bligny Saint Bruno. Le premier chartreux, Rennes, 1984.

Bougard - Noyé 1988 = F. Bougard, G. Noyé, Squillace au Moyen Âge, dans R. Spada (éd.), Da Skylletion a Scolacium. Il parco archeologico della Roccelletta. Mostra di Milano, maggio 1988, Rome-Reggio di Calabria, 1988, p. 215-229.

Brébion $1974=$ Le brébion de la métropole byzantine de Règion (vers 1050), éd. A. Guillou, Cité du Vatican,
S. Stefano del Bosco, a disparu, signe que même l'héritage symbolique s'est éteint. Rappelons cependant que dès la fondation, l'ermitage de S. Maria de Turri devait tout au comte Roger I ${ }^{\mathrm{er}}$, que même les faux documents forgés au XIII ${ }^{\mathrm{e}}$ siècle mettent en avant comme le véritable fondateur ${ }^{77}$. Et si, dès le milieu du XII ${ }^{e}$ siècle, la fondation calabraise de Bruno s'était structurellement muée en un établissement monastique classique, de type bénédictin, inscrit dans les réseaux de pouvoirs, Bruno lui-même, en aidant Roger I ${ }^{\text {er }}$ dans ses prérogatives ecclésiastiques (lorsqu'il le conseille pour la reconstruction de l'évêché de Squillace en 1096), avait revêtu momentanément, avant le retour à la solitude, les habits du saint homme conseiller des grands. Plus que jamais, la "trahison" des successeurs de Bruno, de Lambert (qui fonda S. Stefano del Bosco) à Guillaume de Messine (qui en 1192 accompagna le passage à l'ordre cistercien), apparaît comme une reconstruction moderne.

Annick Peters-Custot

1974 (Corpus des Actes Grecs d'Italie et de Sicile. Recherches d'histoire et de géographie, IV).

Bruno de Cologne en Calabre = A. Peters-Custot, Bruno de Cologne en Calabre, HDR soutenue le 3 décembre 2012 à l'Université de Paris 1, à paraître dans la Collection de l'École française de Rome.

Cahen $1940=$ C. Cahen, Le régime féodal de l'Italie normande, Paris, 1940.

Carocci 2009 = S. Carocci, Angararii e franci. Il villanaggio meridionale, dans E. Cuozzo, J.-M. Martin (éd.), Studi in margine all'edizione della Platea di Luca arcivescovo di Cosenza (1203-1227), Avellino, 2009, p. 205-241.

Catalogus Baronum 1972 = Catalogus Baronum, éd. E. Jamison, Rome, 1972 (Fonti per la storia d'Italia pubblicate dall'Istituto storico italiano per il Medio Evo, 101). 
Cuozzo 1972 = E. Cuozzo, Catalogus Baronum. Commentario, Rome, 1972 (Fonti per la storia d'Italia pubblicate dall'Istituto storico italiano per il Medio Evo, 101).

Cuozzo 1989 = E. Cuozzo, "Quei maledetti Normanni». Cavalieri e organizzazione militare nel Mezzogiorno normanno, Naples, 1989.

Cuozzo - Martin 2009 = E. Cuozzo, J.-M. Martin (éd.), Studi in margine all'edizione della Platea di Luca arcivescovo di Cosenza (1203-1227), Avellino, 2009.

Die Urkunden der Kaiserin Konstanze $1990=$ Die Urkunden der Kaiserin Konstanze, éd. T. Kölzer, Hannovre, 1990 (Monumenta Germaniae Historica. Diplomata Regum et imperatorum Germaniae, XI, III. Constantiae Imperatricis Diplomata).

Dubois 1968 = J. Dubois, Quelques problèmes de l'histoire de l'ordre des chartreux. À propos de livres récents, dans Revue d'histoire ecclésiastique, 63-1, 1968, p. 27-54.

Enzensberger 1981-1982 = H. Enzensberger, Utilitas Regis. Note di storia amministrativa e giuridica e di propaganda politica nell'età dei due Guglielmi, dans Atti della Accademia di Scienze, Lettere e Arti di Palermo, Ser. 5, 1. Parte seconda. Lettre, 1981-1982, p. 23-61.

Enzensberger $1971=\mathrm{H}$. Enzensberger, Beiträge zum Kanzlei - und Urkundenwesen der normannischen Herrscher Unteritaliens und Siziliens, Kallmünz, 1971 (Münchener Historische Studien, Abt. Geschichtliche Hilfswissenschaften, 9).

Falkenhausen 1998a = V. von Falkenhausen, $I$ diplomi dei re normanni in lingua greca, dans G. De Gregorio, O. Kresten (éd.), Documenti medievali greci e latini. Studi comparativi. Atti del seminario di Erice (23-29 octobre 1995), Spolète, 1998 (Centro italiano di Studi sull'alto Medioevo), p. 253-308.

Falkenhausen 1998b = V. von Falkenhausen, Zur Regenschaft der Adelasia del Vasto in Kalabrien und Sizilien (1101-1112), dans AETOS. Studies in honour of Cyril Mango presented to him on April 14. 1998, StuttgartLeipzig, 1998, p. 87-115.

Falkenhausen 2012 = V. von Falkenhausen, Amministrazione fiscale nell'Italia meridionale bizantina (secoli $I X-X I)$, dans J.-M. Martin, A. Peters-Custot, V. Prigent (éd.), L'héritage byzantin en Italie (VIII ${ }^{e}-X I I^{e}$ siècles). II. Droit et institutions publiques, Rome, 2012 (Collection de l'École française de Rome, 461), p. 533-556.

Fodale $1970=$ S. Fodale, Comes et legatus. Sul privilegio di Urbano II e la pretesa Apostolica Legazia dei Normanni di Sicilia, Palerme, 1970 (Università di Palermo. Istituto di Storia medioevale. Studi, 2).

Fodale 1991 = S. Fodale, L'Apostolica Legazia e altri studi su Stato e Chiesa, Messine, 1991.
Guillelmi Regis Diplomata 1996 = Guillelmi Regis Diplomata, éd. H. Enzensberger, Cologne-Vienne, 1996 (Codex Diplomaticus Regni Siciliae. Series I, III).

Historia Diplomatica Friderici secundi 1852-1861 = Historia Diplomatica Friderici secundi, éd. J.-L.-A. HuillardBréholles, 6 tomes en 11 volumes, Paris, 1852-1861, réimpr. anast., Turin, 1963.

Hugues Falcand 1897 = Hugues Falcand, La Historia, 0 Liber de Regno Sicilie, éd. G. B. Siragusa, Rome, 1897 (Fonti per la Storia d'Italia, 22).

Johns 2002 = J. Johns, Arabic Administration in Norman Sicily. The royal dîwân, Cambridge, 2002 (Cambridge Studies in Islamic Civilization).

Kehr 1975 = P.-F. Kehr, Italia Pontificia, X. Calabriainsulae, éd. W. Holtzmann, G. Girgensohn, Zürich, 1975.

La Théotokos de Hagia-Agathè $1972=$ La Théotokos de Hagia-Agathè (Oppido) (1050-1064/1065), éd. A. Guillou, Cité du Vatican, 1972 (Corpus des Actes Grecs d'Italie et de Sicile. Recherches d'histoire et de géographie, III).

Laporte 1960 = Dom Maurice Laporte, Aux sources de la vie cartusienne, I. Éclaircissements concernant la vie de saint Bruno, In Domo Cartusiae, 1960.

Martin 1999 = J.-M. Martin, Aristocraties et seigneuries en Italie méridionale aux XI et XII siècles : essai de typologie, dans Journal des Savants, 1999, p. 227-259.

Martin 2001 = J.-M. Martin, Centri fortificati, potere feudale e organizzazione dello spazio, dans A. Placanica (éd.), La Calabria medievale. I quadri generali, Rome, 2001, p. 487-522.

Martin 2008 = J.-M. Martin, Les seigneuries monastiques, dans R. Licinio, F. Violante (éd.), Nascita di un regno. Poteri signorili, istituzioni feudali e strutture sociali nel Mezzogiorno normanno (1130-1194). Atti delle diciasettesime giornate normanno-sveve (Bari, 10-13 ottobre 2006), Bari, 2008, p. 177-206.

Ménager $1957=$ L.-R. Ménager, Notes et documents sur quelques monastères de Calabre à l'époque normande, dans Byzantinische Zeitschrift, 50, 1957, p. 7-30.

Ménager $1980=$ L.-R. Ménager, Recueil des actes des ducs normands d'Italie [1046-1127]. I. Les premiers ducs (1046-1087), Bari, 1980 (Società di Storia patria per la Puglia. Documenti e monografie, 45).

Ménager 1983 = L.-R. Ménager, Lanfranco, notaio pontificio (1091-1093). La diplomatica ducale italo-normanna e la certosa di S. Stefano del Bosco, dans Studi storici meridionali, 3, 1983, p. 3-35.

MGH 1885 = Monumenta Germaniae Historica, Scriptores, XXVII, Hannovre, 1885.

Nef $2000=\mathrm{A}$. Nef, Conquêtes et reconquêtes médiévales: 
une réduction en servitude généralisée? (Al-Andalus, Sicile et Orient latin), dans Les formes de la servitude: esclavages et servages de la fin de l'Antiquité au monde moderne. Actes de la table ronde de Nanterre (12-13 décembre 1997), dans Mélanges de l'École française de Rome. Moyen Âge, 112-2, 2000, p. 579-607.

Nef 2011 = A. Nef, Conquérir et gouverner la Sicile islamique aux $X I^{e}$ et $X I I^{e}$ siècles, Rome, 2011 (BEFAR, 346).

Patlagean 2008 = E. Patlagean, Les hommes (anthrôpoi) dans les documents grecs du Mezzogiorno normand, dans E. Cuozzo, V. Déroche, A. Peters-Custot, V. Prigent (éd.), Puer Apuliae, Mélanges en l'honneur de JeanMarie Martin, 2 vol. , Paris, 2008 (Centre de recherche d'histoire et de civilisation de Byzance. Monographies, 30), II, p. 529-536.

Peters-Custot $2007=$ A. Peters-Custot, Les remaniements de la carte diocésaine de l'Italie grecque lors de la conquête normande: une politique de latinisation forcée de l'espace? (1059-1130), dans P. Rodriguez (éd.), Pouvoir et territoire, Colloque du CERHI, Saint-Étienne, 7-8 novembre 2005, Saint-Étienne, 2007 (Travaux du CERHI, 6), p. 57-77.

Peters-Custot $2008=$ A. Peters-Custot, Brébion, kodex et plateae : petite enquête sur les instruments de la propriété monastique dans la Calabre méridionale aux époques byzantine et normande, dans E. Cuozzo, V. Déroche, A. Peters-Custot, V. Prigent (éd.), Puer Apuliae, Mélanges en l'honneur de Jean-Marie Martin, 2 vol. , Paris, 2008 (Centre de recherche d'histoire et de civilisation de Byzance. Monographies, 30), II, p. 537-552.

Peters-Custot $2009=$ A. Peters-Custot, Les Grecs de l'Italie méridionale post-byzantine. Une acculturation en douceur (IX ${ }^{e}$-XIV s.), Rome, 2009 (Collection de l'École française de Rome, 420).

Peters-Custot 2012a = A. Peters-Custot, Plateae et anthrôpoi, peut-on trouver des origines byzantines à l'organisation normande de la paysannerie de la Calabre méridionale?, communication présentée lors de la quatrième session du programme L'héritage byzantin en Italie, VIII ${ }^{e}-X I I^{e}$ siècle. Les structures rurales, École française de Rome, 17-18 décembre 2010 (à paraître).

Peters-Custot 2012b = A. Peters-Custot, Les plateae calabraises d'époque normande, une source pour l'histoire économique et sociale de la Calabre byzantine?, à paraître dans les Cahiers de recherches médiévales et humanistes, 2012.

Pirri 1733 = R. Pirri, Sicilia Sacra, I, Palerme, 1733, réimpr. anast. Bologne, 1987.

Regii Neapolitani Archivi 1861 = Regii Neapolitani Archivi Monumenta, VI (1115-1130), Appendice 11, Naples, 1861.

Rogerii II. 1987 = Rogerii II. Regis diplomata latina, éd. C. Brühl, Cologne-Vienne, 1987 (Codex Diplomaticus Regni Siciliae. Series I, II, 1).

Rognoni $2004=\mathrm{C}$. Rognoni, Les actes privés grecs de l'Archivio Ducal de Medinaceli (Tolède), I., Les monastères de Saint-Pancrace de Briatico, de San Filippo de Bojôannès, et de S. Nicola-des-Drosi (Calabre, XI'-XII siècles), Paris, 2004.

Saint-Jean-Théristès $1980=$ Saint-Jean-Théristès (10541264), éd. S. G. Mercati, C. Giannelli, A. Guillou, Cité du Vatican, 1980 (Corpus des Actes Grecs d'Italie et de Sicile. Recherches d'histoire et de géographie, V).

Schlichte 2005 = A. Schlichte, Der «gute» König. Wilhelm II. von Sizilien (1166-1189), Tübingen, 2005 (Bibliothek des deutschen historischen Instituts in Rom, 110).

Storia critico-cronologica diplomatica 1775 = Storia criticocronologica diplomatica del patriarca S. Brunone e del suo ordine cartusiano compilata dal P. D. Benedetto Tromby, Naples, 1775 réimpr. anast. Salzbourg, 1982 (Analecta Cartusiana, 84).

Tancredi et Willelmi III 1982 = Tancredi et Willelmi III regum diplomata, éd. H. Zielinski, Cologne-Vienne, 1982 (Codex Diplomaticus Regni Siciliae. Series I, V).

Taviani-Carozzi $2003=$ H. Taviani-Carozzi, Saint Bruno en Calabre, entre politique normande et projet pontifical, dans A. Girard, D. Le Blevec, N. Nabert (éd.), Saint Bruno et sa postérité spirituelle. Actes du colloque international des 8 et 9 octobre 2001. Institut catholique de Paris, Salzbourg, 2003 (Analecta Cartusiana, 189), p. 59-79.

Toomaspoeg $2009=\mathrm{K}$. Toomaspoeg, Decimae. Il sostegno economico dei sovrani alla Chiesa del Mezzogiorno nel XIII secolo. Dai lasciti di Eduard Sthamer e Norbert Kamp, Rome, 2009 (Ricerche dell'Istituto storico germanico di Roma, 4).

Trinchera 1865 = F. Trinchera, Syllabus graecarum membranarum..., Naples, 1865. 